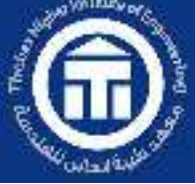

INTERNATIONAL JOURNAL OF ADVANCED ENGINEERING

AND BUSINESS SCIENCES (IJAEBS)

Journal homepage: International Journal of Advanced Engineering and Business Sciences (ekb.eg)

\title{
Cerium Oxide Nano-Particles Application for Self-Cleaning Building Material
}

\author{
Mai. M. El-Masry ${ }^{a^{*}, \text { Yousra M. Hamdy }}$ \\ $a^{*}$ Basic science Dept., Higher Engineering Institute, Thebes Academy, Cairo, Egypt \\ ${ }^{b}$ Civil Engineering Dept., Higher Engineering Institute, Thebes Academy, Cairo, Egypt \\ *Corresponding author: Email address: mai.elmasry@ thebes.edu.eg
}

Received: 27 April 2021

Accepted: 29 April 2021

Published:30 April 2021

\begin{abstract}
Photocatalytic degradation has gained attraction in the self-cleaning construction materials applications because of its ability to be used to protect surface of cementitious materials and minimize the negative effects of environmental conditions on building, and to remove the dyes and other organic pollutants from water and wastewater. In this study, the influence of nanoCeria particles on the photocatalytic degradation of methylene blue dye of cement mortar samples was investigated. In this work, nanoceria was prepared via simple co-precipitation method. Nanoceria is a powerful photocatalyst due to its high visible-light absorptivity, strong redox ability, high oxygen storage/release capacity, eco-friendly nature, photostability and cost effectiveness. Nano ceria showed a photocatalytic and self-cleaning activity in the presence of sun radiation. Nano ceria particles were characterized by X-ray diffraction, High resolution transmission electron microscope, and Ultra-violet visible spectroscopy. The presence of active oxygen species detected on nano- ceria surface was identified as cause of the photobleaching of dyes in presence of UV-irradiated.
\end{abstract}

Keywords: Photocatalytic, Self- Cleaning Building materials, Ceria, UV, Methylene Blue, photodegradation.

International Journal of Advanced Engineering and Business Sciences, Volume 2, Issue 1, April 2021 - ISSN $2682-2938$ Innovation and Research Center, Faculty of Engineering and Management Science, Integrated Thebes Academy (ITA). 


\section{INTRODUCTION}

Cement mortar or concrete is the predominant building material of most building construction. The exposure of specific environmental conditions leads to changes on the appearance and also leads to physical degradation or corrosion.

Photocatalytic degradation is one of the effective methods to protect surface of cementitious materials and minimize the negative effects of environmental conditions on building, and to remove the dyes and other organic pollutants from water and wastewater.

Over the last few decades, self-cleaning building materials [1] have been developed to overcome the different environmental conditions that leads to change in the building appearance and to physical degradation.

Several photocatalytic nanomaterials exhibit a promising ability to achieve self-cleaning effect of cement mortar or concrete under visible light or UV light [2], [3]. The most common materials are nano titanium [4] and nano zinc-based photocatalytic concrete [5], [6], [7].

Nanoceria, the most abundant rare earth metal oxide $\mathrm{CeO}_{2}$, is a powerful photocatalyst for environmental sustainability due to its high visible-light absorptivity, strong redox ability, high oxygen storage/release capacity, eco-friendly nature, photostability and cost effectiveness [8].

In this paper, the photocatalytic activity of nano-ceria was evaluated by degradation of Methylene Blue dye in the presence of solar radiation as a light source.

The ability of ceria to be easily reduced to nonstoichiometric oxides is related to the properties of fluorite structured mixed valence oxides to deviate from stoichiometry [8].

In the nanoscale, the surface area of cerium oxide particles is dramatically increased [9] which not only increases oxygen exchange but also makes it easy for redox reactions.

The synthesis and thermodynamic conditions used to achieve ultra-small ceria nanoparticles (NPs) produce oxygen deficient ceria crystals.

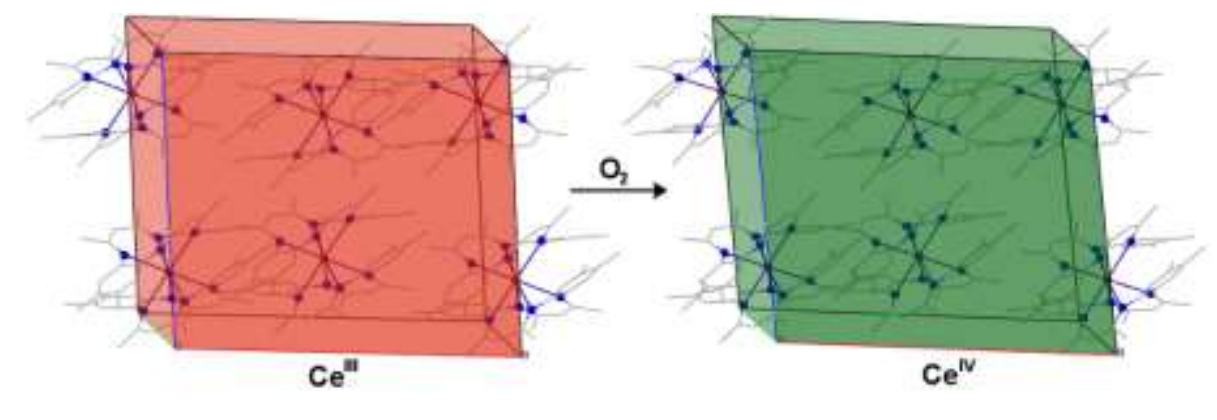

Mai M. El-Masry and Yousra M. Hamdy, IJAEBS (2021), (2), (1), (1-10) 
After $\mathrm{CeO}_{2}$ loses considerable quantities of oxygen from its lattice and forms several oxygen vacancies, the sub oxides are readily reoxidized to $\mathrm{CeO}_{2}$ on exposure to an oxidizing environment. Reduced ceria results from the removal of $\mathrm{O}^{2-}$ ions from the $\mathrm{CeO}_{2}$ lattice [10], which generates an anion vacant site according to the following equations [10]:

$$
\begin{aligned}
\mathrm{Ce}^{4+}+\mathrm{e}^{-} & \leftarrow \mathrm{Ce}^{3+} \\
\mathrm{Ce}^{3+}+\mathrm{O}_{2} & \rightarrow \mathrm{Ce}^{4+}+\mathrm{O}^{-2}
\end{aligned}
$$

In this work, the photocatalytic ability of nanoceria is being applied to test the degradation of methylene blue dye stain on cement mortar sample.

\section{Materials and Experiments}

\subsection{Sample preparation}

\subsubsection{Ceria nanoparticles preparation}

Cerium (III) nitrate $\left(\mathrm{Ce}\left(\mathrm{NO}_{3}\right)_{3} \cdot 6 \mathrm{H}_{2} \mathrm{O}\right)$ was chosen as the precursor to prepare ceria samples by a co-precipitation method. Some drops of ammonium hydroxide $\mathrm{NH}_{4} \mathrm{OH} 33 \%$ were added to

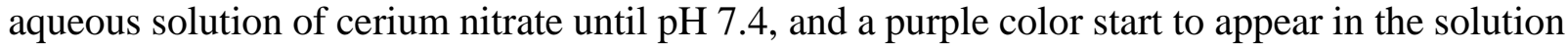
turned in few minutes to a yellowish white precipitation. The collected precipitated cerium oxide nanoparticles were washed several times with deionized water, methanol and ethanol. The precipitated particles were separated using a centrifuge (3500 rpm for 45 minutes). A yellowish white powder of cerium oxide was obtained by drying the collected materials at $70^{\circ} \mathrm{C}$ for $2 \mathrm{hr}$.

\subsubsection{Cement mortar sample preparation}

The cement mortar paste was prepared by normal Portland cement and fine aggregates (sand) in a 1:3 ratio, respectively, and water to cement ratio was about 0.5 .

Two samples were prepared for testing: one control sample without nanoparticles and one sample with added nanoceria as a surface coating. Samples were allowed to cure for 24 hours.

\subsection{Sample Characterization}

\subsubsection{X-ray diffraction (XRD)}

Proker $\mathrm{D}_{8}$ advance $\mathrm{X}$-ray diffractometer with $\mathrm{CuK} \alpha$ radiation $(\lambda=1.5418 \AA)$ has been used to assure the preparation of the cerium oxide in single phase. X-ray diffraction pattern was recorded at room temperature in a wide range of Bragg angles $2 \theta\left(20^{\circ} \leq 2 \theta \leq 80^{\circ}\right)$ with $0.02^{\circ}$ step size.

Mai M. El-Masry and Yousra M. Hamdy, IJAEBS (2021), (2), (1), (1-10) 
From XRD analysis the crystallite size was calculated according to Scherrer's equation as follows $[18,19]$ :

$$
\mathrm{D}=\frac{\mathrm{K} \cdot \lambda}{\beta \cdot \cos \theta}
$$

Where, $D$ is the crystallite size $(\mathrm{nm}), \mathrm{K}$ is the particle shape factor, taken as 0.94 for cerium oxide [20], $\lambda$ is the target wavelength (nm), $\beta$ is the corrected full-width at half maximum, and $\theta$ is the position (angle) of the peak at the maximum.

\subsubsection{High resolution Transmission Electron Microscope (HRTEM)}

The prepared samples were micro graphed and analyzed using (JEOL-1010 model). The dispersive media of the samples were distilled water. The powder was ultra-sonically, dispersed, then deposited on a carbon-coated copper grid and left to dry before examination.

\subsubsection{Ultra-Violet - Visible spectroscopy}

(UV-Vis.) spectroscopic analysis was carried out for all the investigated samples using (Cary 5000 UV-Vis-NIR Spectrophotometer, wavelength range from 175 to 3300nm, Version 1.12, double beam mode). The wavelength considered range is from $200 \mathrm{~nm}$ to $800 \mathrm{~nm}$ with (UV-Vis) scan rate $200 \mathrm{~nm} / \mathrm{min}$, and data interval $1.0 \mathrm{~nm}$ and (UV-Vis.) average time $0.3 \mathrm{sec}$.

\subsection{Photocatalytic Evaluation}

The photocatalytic and self-cleaning effect of nanoceria was evaluated by degradation of Methylene Blue dye stain (aqueous solution) on the sample surface with the exposure to direct sun light as a source of UV radiation for 4 hours.

Ceria nano particles were dispersed in water to make a slurry and used as a photocatalyst to the cement mortar sample by coating its surface. Few drops of MB aqueous solution were applied on the two prepared cement samples. Then, both samples were exposed to the sun radiation as a source of UV radiation.

The effect of nano ceria to decompose the MB dye has been visually observed and photographed every hour. 


\section{RESULTS and DISCUSSION}

\subsection{Particle size measurements}

\subsubsection{XRD}

The XRD pattern of the prepared nanoparticles is shown in Fig. (1). Perfect identification of the peaks of (111), (200), (220), and (311) are confirmed the presence of the investigated sample in a single crystalline phase of $\mathrm{CeO}_{2}$. The crystallite size was calculated from X-ray data using Scherrer's equation. The crystallite size was $17.8 \mathrm{~nm}$.

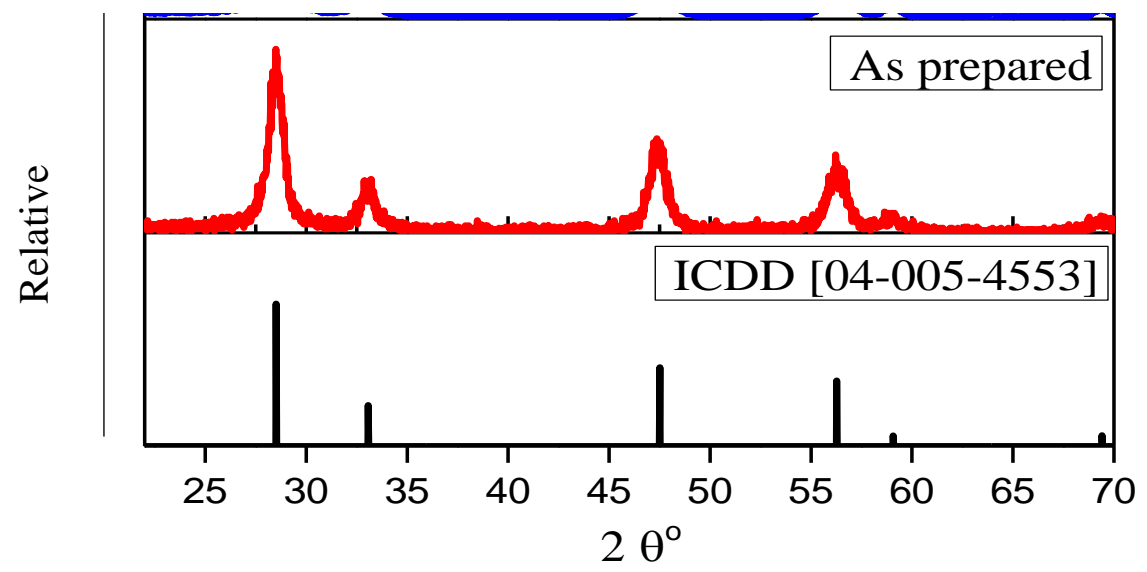

Fig. (1) XRD spectra of the Ceria prepared

\subsubsection{HRTEM}

HRTEM micrographs of the prepared $\mathrm{CeO}_{2}$ sample is shown in Fig. (2), where a homogenous distribution of polycrystalline nanometer particles was appeared. It is noted that the considered preparation technique was succeeded to produce ceria nanoparticles of about $12 \mathrm{~nm}$. 


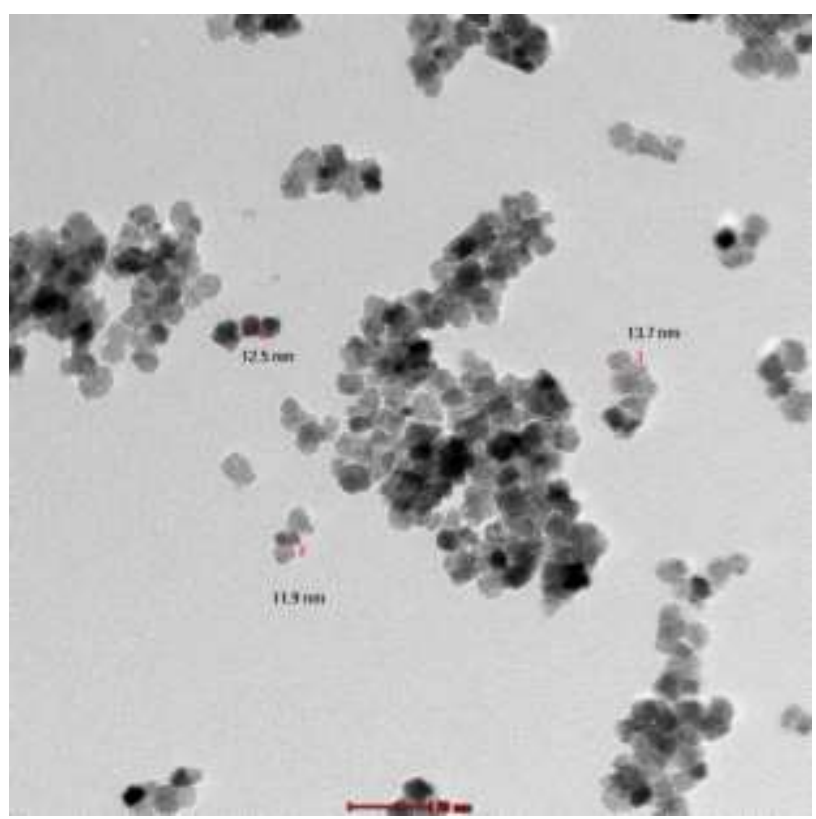

Fig. (2) HRTEM micrographs of the Ceria prepared

\subsection{3 (UV-vis.) spectrophotometry}

(UV- vis.) spectrophotometry of cerium is of particular interest as it provides quantitative information about the type of oxidation state and the reaction rate. In previously published data [11], [12] [13], smaller particles have shown a red-shifted band gap. The reason that ceria nanoparticles have an opposite effect to that observed in other semiconducting nanoparticles. This is not back to a quantum mechanical phenomenon, but rather that, as the particles get smaller, they have a higher ratio of $\mathrm{Ce}^{3+} / \mathrm{Ce}^{4+}$. This increase in $\mathrm{Ce}^{3+}$ ions within the crystal lattice causes a red shift in the absorbance of the material; attributable to the defect states associated with the $\mathrm{Ce}^{3+}$ ions and oxygen vacancies [14]. The defect states reside $3 \mathrm{eV}$ above the $\mathrm{CeO}_{2}$ valence band. The $\mathrm{CeO}_{2}$, containing $\mathrm{Ce}^{4+}$ ions, has a band gap of $4 \mathrm{eV}$. As the concentration of $\mathrm{Ce}^{3+}$ ions increase, the concentration of $\mathrm{Ce}^{4+}$ ions decreases and more absorption will occur at $3 \mathrm{eV}$ and less at $4 \mathrm{eV}$ [15] [16], causing a redshift in the absorption spectrum. Fig. (3) illustrates the (UV- vis.) spectra for the prepared sample. The data showed that the samples reveal maximum UV absorbance peak at wavelengths $(302 \mathrm{~nm})$ when dispersed in water and ethylene glycol, respectively. Which makes nanoceria a good UV absorber in different applications. 


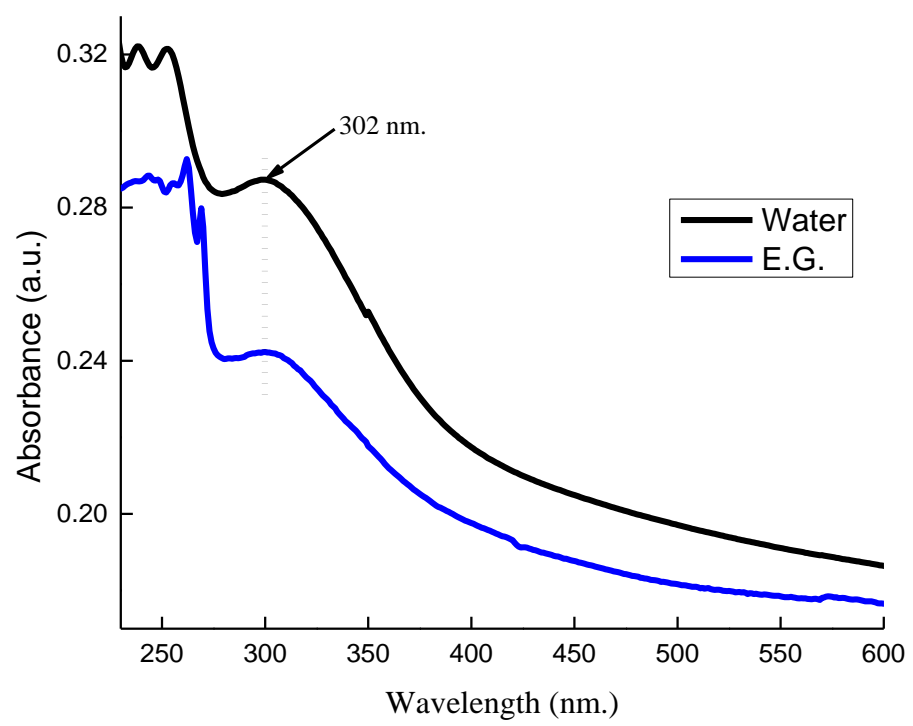

Fig. (3) (UV-vis.) absorbance spectra of the Ceria prepared sample dispersed in both water and E.G.

\subsection{Nano ceria as self-cleaning building material}

The change of the Methylene blue color stain on the cement samples during exposure to direct sun light for 4 hours is shown in Fig. (4). It was observed that MB color on the nanoceriacement sample was gradually degrading, while there was just a slight discoloration of MB dye in the pure cement mortar sample (without Ceria). This confirms the role of ceria nanoparticles which act as a photocatalyst materials. It can be clearly seen that the MB color starts degrading after $60 \mathrm{~min}$ of exposing to sunlight and after $240 \mathrm{mins}$ (4 hrs), a significant discoloration was observed. Also, it is evident from Fig. (4) that the degradation color of MB is proportional with time and that the degradation level was increased with time, on the cement mortar surface. In general, the presence of nanoceria particles induced the self-cleaning properties of the cement sample. 

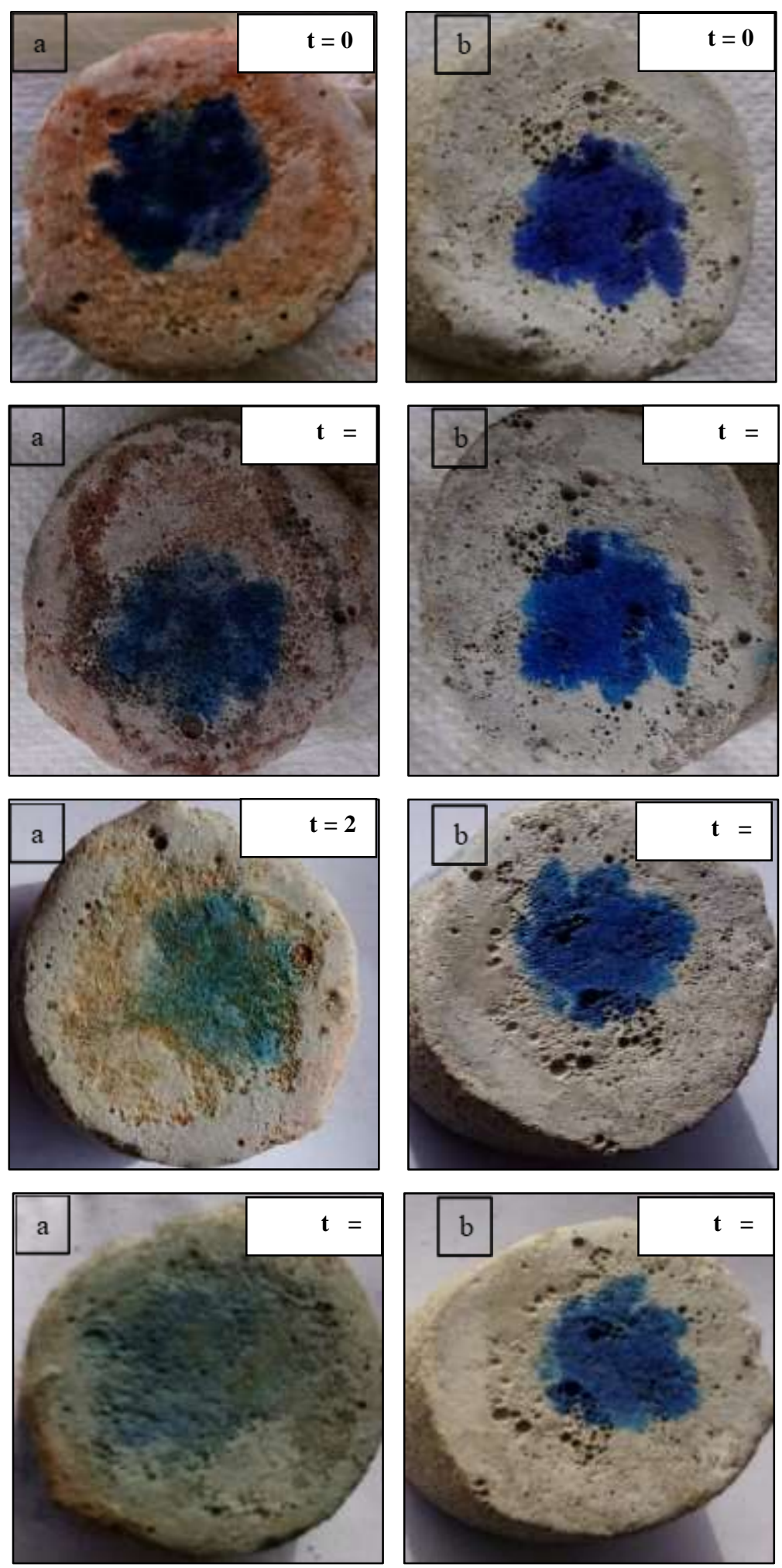

Fig. (4) Recorded photos of the cement samples with (a)nano-ceria-cement samples dispersed in water and (b) pure cement mortar sample.

Mai M. El-Masry and Yousra M. Hamdy, IJAEBS (2021), (2), (1), (1-10) 


\section{CONCLUSION}

Nanoceria coating was applied on a cement sample as a photocatalyst to achieve photodegradation of methylene blue dye in visible light region. Discoloration of the dye on the nanoceria-cement-stained sample was achieved after couple of hours of direct sunlight exposure. It was illustrated by visual observations that nanoceria can be an effective photocatalytic and self-cleaning effect for cementitious and concrete materials.

\section{REFERENCES}

[1] J. Y. E.-H. Y. Aiqin Zhao, "Self-cleaning engineered cementitious composites," Cement and Concrete Composites, vol. 64, pp. 74-83, 2015.

[2] L. M. L. I. G. R. D. D. W. B. Arsou Arimi, "Visible-light photocatalytic activity of zinc ferrites," Chemistry, vol. 366, pp. 118-126, 2018.

[3] J. Z. A. C. K. B. K.-N. A. W. M. Magdalena Janusa, "Self-cleaning properties of cement plates loaded with N, C- modified TiO2 photocatalysts," Applied Surface Science, vol. 330, pp. 200-206, 2015.

[4] C. H. a. Y. C. Zhan Guo, "Experimental study on photocatalytic degradation efficiency of mixed crystal nano-TiO2 concrete.," Nanotechnol. Rev, vol. 9, pp. 219$229,2020$.

[5] A. B. N. M. M. M. A. B. A. Siti Norsaffirah Zaila, "Influence of ZnO Nanoparticles on Mechanical Properties and Photocatalytic Activity of Self-cleaning ZnO-Based Geopolymer Paste.," Journal of Inorganic and Organometallic polymers and materials, vol. 30, 2020.

[6] J. V. S. d. M. Bruno Oliveira Bica, "Concrete blocks nano-modified with zinc oxide $(\mathrm{ZnO})$ for photocatalytic paving: Performance comparison with titanium dioxide (TiO2)," Construction and Building Materials, vol. 252, pp. 119-120.

[7] F. M. ,. S. T. F. A. R. Fatemeh Ajormal, "Zinc Ferrite Nanoparticles in PhotoDegradation of Dye," Journal of Chemical Reviews, vol. 2, pp. 10-90, 2020. 
[8] J. A. K. S. D. Radhika R. Nair, "Ceria doped titania nano particles: Synthesis and photocatalytic activity,", Materials Today: Proceedings, vol. 3, no. 6, pp. 1643-1649, 2016.

[9] I. M. L. Baile Wu, "Surface Functional Group Engineering of CeO2 Particles for Enhanced Phosphate Adsorption," Environmental Science \& Technology, vol. 54, no. 7, p. 4601-4608, 2020.

[10] D. C. a. S. L. Adnan Younis, Cerium Oxide Nanostructures and their Applications, IntechOpen, 2016.

[11] H. L. H. Z. Z. W. L. C. Chunwen Sun, "Controlled synthesis of CeO2 nanorods by a solvothermal method," Nanotechnology, vol. 16, no. 9, p. $1454,2005$.

[12] J.-T. Y. K. A. K. Shin Tsunekawa, "Blue shifts in the ultraviolet absorption spectra of cerium oxide nanocrystallites," JOURNAL OF APPLIED PHYSICS, vol. 94, no. 5, p. $3654,2003$.

[13] P. K. K. R. L. M. G. L. K. K. J. Stoimenov, "Metal Oxide Nanoparticles as Bactericidal Agents.," Langmuir., vol. 18, p. 6679, 2002.

[14] M. R. J. W. R. J. F. T. K. Hassellöv, "Nanoparticle analysis and characterization methodologies in environmental risk assessment of engineered nanoparticles.," Ecotoxicology., vol. 17, p. 344, 2008.

[15] A. Trovarelli, Catalysis by Ceria and Related Materials, vol. 68, World Scientific, 2013, p. 035104.

[16] S. d. G. S. B. S. V. G. a. B. G. Fabris, "Taming multiple valency with density functionals: A case of defective ceria," Physical review, vol. 71, no. 4, p. 041102, 2005.

[17] C. B. C. David Bernard Williams, Transmission Electron Microscopy: A Textbook for Materials Science. Diffraction. II, 1996.

[18] R. A. Fava, Crystal Structure and Morphology, 1980.

[19] T. F. Nollet L. M. L., "Handbook of Analysis of Active Compounds in Functional Foods", CRC Press , 2012. 